\title{
On the uniqueness of the solution of the two-dimensional Navier-Stokes equation with a Dirac mass as initial vorticity
}

\author{
Isabelle Gallagher \\ Université Paris 7 \\ Institut de Mathématiques de Jussieu \\ Case 7012, 2 place Jussieu \\ 75251 Paris Cedex 05 France \\ Pierre-Louis Lions \\ CEREMADE \\ Université de Paris-Dauphine \\ 75775 Paris cedex 16, France
}

Thierry Gallay

Institut Fourier

Université de Grenoble I

38402 Saint-Martin d'Hères, France

\begin{abstract}
We propose two different proofs of the fact that Oseen's vortex is the unique solution of the two-dimensional Navier-Stokes equation with a Dirac mass as initial vorticity. The first argument, due to C.E. Wayne and the second author, is based on an entropy estimate for the vorticity equation in self-similar variables. The second proof is new and relies on symmetrization techniques for parabolic equations.
\end{abstract}

\section{Introduction}

We consider the vorticity equation associated to the two-dimensional Navier-Stokes equation, namely

$$
\partial_{t} \omega(x, t)+u(x, t) \cdot \nabla \omega(x, t)=\Delta \omega(x, t), \quad x \in \mathbf{R}^{2}, \quad t>0 .
$$

The velocity field $u(x, t) \in \mathbf{R}^{2}$ is obtained from the vorticity $\omega(x, t) \in \mathbf{R}$ via the Biot-Savart law

$$
u(x, t)=\frac{1}{2 \pi} \int_{\mathbf{R}^{2}} \frac{(x-y)^{\perp}}{|x-y|^{2}} \omega(y, t) \mathrm{d} y, \quad x \in \mathbf{R}^{2}, \quad t>0
$$

where $\left(x_{1}, x_{2}\right)^{\perp}=\left(-x_{2}, x_{1}\right)$. It satisfies $\operatorname{div} u=0$ and $\partial_{1} u_{2}-\partial_{2} u_{1}=\omega$. Equations (1.1), (1.2) are invariant under the scaling transformation

$$
\omega(x, t) \mapsto \lambda^{2} \omega\left(\lambda x, \lambda^{2} t\right), \quad u(x, t) \mapsto \lambda u\left(\lambda x, \lambda^{2} t\right), \quad \lambda>0
$$

The Cauchy problem for the vorticity equation (1.1) is globally well-posed in the (scale invariant) Lebesgue space $L^{1}\left(\mathbf{R}^{2}\right)$, see for instance [6]. To include more general initial data, such as isolated vortices or vortex filaments, it is necessary to use larger function spaces. A natural candidate is the space $\mathcal{M}\left(\mathbf{R}^{2}\right)$ of all finite real measures on $\mathbf{R}^{2}$, equipped with the total variation norm. This space contains $L^{1}\left(\mathbf{R}^{2}\right)$ as a closed subspace, and its norm is invariant under (the spatial part of) the rescaling (1.3). Moreover, $\mathcal{M}\left(\mathbf{R}^{2}\right)$ is closed with respect to the following weak convergence: $\mu_{n} \rightarrow \mu$ if $\int_{\mathbf{R}^{2}} \varphi \mathrm{d} \mu_{n} \rightarrow \int_{\mathbf{R}^{2}} \varphi \mathrm{d} \mu$ for any continuous function $\varphi: \mathbf{R}^{2} \rightarrow \mathbf{R}$ vanishing at infinity. 
Existence of solutions of (1.1) with initial data in $\mathcal{M}\left(\mathbf{R}^{2}\right)$ was first proved by Cottet 10, and independently by Giga, Miyakawa and Osada [13, see also Kato [15. Uniqueness can be obtained by a standard Gronwall argument if the atomic part of the initial vorticity $\mu$ is sufficiently small [13, 15], but this method is bound to fail if $\mu$ contains large Dirac masses. In the particular case where $\mu=\alpha \delta_{0}$ for some $\alpha \in \mathbf{R}$, an explicit solution is known:

$$
\omega(x, t)=\frac{\alpha}{t} G\left(\frac{x}{\sqrt{t}}\right), \quad u(x, t)=\frac{\alpha}{\sqrt{t}} v^{G}\left(\frac{x}{\sqrt{t}}\right), \quad x \in \mathbf{R}^{2}, \quad t>0,
$$

where

$$
G(\xi)=\frac{1}{4 \pi} \mathrm{e}^{-|\xi|^{2} / 4}, \quad v^{G}(\xi)=\frac{1}{2 \pi} \frac{\xi^{\perp}}{|\xi|^{2}}\left(1-\mathrm{e}^{-|\xi|^{2} / 4}\right), \quad \xi \in \mathbf{R}^{2}
$$

This self-similar solution of the two-dimensional Navier-Stokes equation is often called the LambOseen vortex with total circulation $\alpha$. It is the unique solution with initial vorticity $\alpha \delta_{0}$ in the following precise sense:

Theorem 1.1 [12] Let $T>0, K>0, \alpha \in \mathbf{R}$, and assume that $\omega \in C^{0}\left((0, T), L^{1}\left(\mathbf{R}^{2}\right) \cap\right.$ $\left.L^{\infty}\left(\mathbf{R}^{2}\right)\right)$ is a solution of (1.1) satisfying $\|\omega(\cdot, t)\|_{L^{1}} \leq K$ for all $t \in(0, T)$ and $\omega(\cdot, t) \rightarrow \alpha \delta_{0}$ as $t \rightarrow 0+$. Then

$$
\omega(x, t)=\frac{\alpha}{t} G\left(\frac{x}{\sqrt{t}}\right), \quad x \in \mathbf{R}^{2}, \quad t \in(0, T) .
$$

Here and in the sequel, we say that $\omega \in C^{0}\left((0, T), L^{1}\left(\mathbf{R}^{2}\right) \cap L^{\infty}\left(\mathbf{R}^{2}\right)\right)$ is a (mild) solution of (1.1) if the associated integral equation

$$
\omega\left(\cdot, t_{2}\right)=\mathrm{e}^{\left(t_{2}-t_{1}\right) \Delta} \omega\left(\cdot, t_{1}\right)-\int_{t_{1}}^{t_{2}} \nabla \cdot \mathrm{e}^{\left(t_{2}-t\right) \Delta}(u(\cdot, t) \omega(\cdot, t)) \mathrm{d} t
$$

is satisfied for all $0<t_{1}<t_{2}<T$.

The particular case covered by Theorem 1.1 is important for at least two reasons. First, it has deep connections with the long-time behavior of smooth solutions. Indeed, if $\omega(x, t)$ is any solution of (1.1) such that $\omega(\cdot, t) \in L^{1}\left(\mathbf{R}^{2}\right)$ and $\int_{\mathbf{R}^{2}} \omega(x, t) \mathrm{d} x=\alpha$ for all $t>0$, it is shown in [12] that $\omega(\cdot, t)$ converges in $L^{1}\left(\mathbf{R}^{2}\right)$ to the Oseen vortex with circulation $\alpha$ as $t \rightarrow+\infty$. This convergence result can in fact be deduced from Theorem 1.1 by a classical renormalization argument, see [9] and [14. On the other hand, combining the standard Gronwall argument (which works if the initial measure has small atomic part) with Theorem 1.1 (which allows to treat a large Dirac mass), it is possible to prove the uniqueness of the solution of (1.1) for arbitrary data $\mu \in \mathcal{M}\left(\mathbf{R}^{2}\right)$ [1]. Thus, the Cauchy problem for (1.1) is globally well-posed in the space $\mathcal{M}\left(\mathbf{R}^{2}\right)$ too.

The proof of Theorem 1.1] in [12] is based on an entropy estimate for equation (1.1) rewritten in self-similar variables. For completeness, this argument will be reproduced below in its simplest form. The real purpose of this paper is to provide an alternative proof of Theorem 1.1 which relies on completely different ideas: it is based on symmetrization techniques for elliptic and parabolic equations which originate in the work of Talenti [18, see [2, 3]. These methods ultimately rely on the maximum principle, whereas the original proof of [12] uses a Lyapunov function. We find the comparison of both arguments very instructive, and this is why we chose to collect them in the present article.

The rest of the text is organized as follows. Under the assumptions of Theorem [1.1, we first derive a priori estimates on the solution $\omega(x, t)$ using Gaussian bounds on the fundamental solution of the convection-diffusion equation, which are due to Osada in [17. This preliminary part is common to both approaches. Following [12], we next introduce the self-similar 
variables $\xi=x / \sqrt{t}, \tau=\log (t)$ and we show that the rescaled vorticity $w(\xi, \tau)=\mathrm{e}^{\tau} \omega\left(\xi \mathrm{e}^{\frac{\tau}{2}}, \mathrm{e}^{\tau}\right)$ satisfies an entropy estimate which implies the conclusion of Theorem 1.1. The new proof begins in Section 4, where we recall the definition of the symmetric nonincreasing rearrangement of a function. In particular, we prove that the solution of a convection-diffusion equation of the form (1.1) with initial data $\omega_{0}$ is "dominated" (in a sense to be precised) for all times by the solution of the heat equation with symmetrized initial data $\omega_{0}^{\#}$. In the final section, we show how this key estimate provides a new and short proof of Theorem 1.1.

\section{Representation and a priori estimates}

Assume that $\omega \in C^{0}\left((0, T), L^{1}\left(\mathbf{R}^{2}\right) \cap L^{\infty}\left(\mathbf{R}^{2}\right)\right)$ is a solution of (1.1) satisfying the hypotheses of Theorem [1.1 From [7] we know that $\omega(x, t)$ coincides for $t>0$ with a classical solution of (1.1) in $\mathbf{R}^{2}$ as constructed for instance in [6]. In particular $\omega(x, t)$ is smooth for $t>0$. Since the Cauchy problem for (1.1) is globally well-posed in $L^{1}\left(\mathbf{R}^{2}\right) \cap L^{\infty}\left(\mathbf{R}^{2}\right)$ and since the $L^{1}$ norm of $\omega(\cdot, t)$ is nonincreasing with time, we can assume that $T=+\infty$ without loss of generality. By assumption $\|\omega(\cdot, t)\|_{L^{1}} \leq K$ for all $t>0$, hence the associated velocity field satisfies $t^{\frac{1}{2}}\|u(\cdot, t)\|_{L^{\infty}} \leq C K$ for all $t>0$, where $C>0$ is a universal constant, see ([8], Theorem 2).

The solution $\omega(x, t)$ of (1.1) has an integral representation of the form

$$
\omega(x, t)=\int_{\mathbf{R}^{2}} \Gamma_{u}(x, t ; y, s) \omega(y, s) \mathrm{d} y, \quad x \in \mathbf{R}^{2}, \quad t>s>0,
$$

where $\Gamma_{u}$ is the fundamental solution of the convection-diffusion equation $\partial_{t} \omega+u \cdot \nabla \omega=\Delta \omega$. The following properties of $\Gamma_{u}$ are due to Osada [17] and to Carlen and Loss [8].

- For any $\beta \in(0,1)$ there exists $K_{1}>0$ (depending only on $K$ and $\beta$ ) such that

$$
0<\Gamma_{u}(x, t ; y, s) \leq \frac{K_{1}}{t-s} \exp \left(-\beta \frac{|x-y|^{2}}{4(t-s)}\right)
$$

for all $x, y \in \mathbf{R}^{2}$ and $t>s>0$, see [8]. A similar Gaussian lower bound is also known.

- There exists $\gamma \in(0,1)$ (depending only on $K$ ) and, for any $\delta>0$, there exists $K_{2}>0$ (depending only on $K$ and $\delta$ ) such that

$$
\left|\Gamma_{u}(x, t ; y, s)-\Gamma_{u}\left(x^{\prime}, t^{\prime} ; y^{\prime}, s^{\prime}\right)\right| \leq K_{2}\left(\left|x-x^{\prime}\right|^{\gamma}+\left|t-t^{\prime}\right|^{\gamma / 2}+\left|y-y^{\prime}\right|^{\gamma}+\left|s-s^{\prime}\right|^{\gamma / 2}\right),
$$

whenever $t-s \geq \delta$ and $t^{\prime}-s^{\prime} \geq \delta$, see [17].

- For $t>s>0$ and $x, y \in \mathbf{R}^{2}$,

$$
\int_{\mathbf{R}^{2}} \Gamma_{u}(x, t ; y, s) \mathrm{d} x=1, \quad \int_{\mathbf{R}^{2}} \Gamma_{u}(x, t ; y, s) \mathrm{d} y=1 .
$$

If $x, y \in \mathbf{R}^{2}$ and $t>0$, it follows from (2.3) that the function $s \mapsto \Gamma_{u}(x, t ; y, s)$ can be continuously extended up to $s=0$, and that this extension (still denoted by $\Gamma_{u}$ ) satisfies properties (2.2) to (2.4) with $s=0$. Using this observation, we obtain for all $x \in \mathbf{R}^{2}$ and all $t>0$

$$
\begin{aligned}
\omega(x, t) & =\int_{\mathbf{R}^{2}} \Gamma_{u}(x, t ; y, 0) \omega(y, s) \mathrm{d} y \\
& +\int_{\mathbf{R}^{2}}\left(\Gamma_{u}(x, t ; y, s)-\Gamma_{u}(x, t ; y, 0)\right) \omega(y, s) \mathrm{d} y, \quad 0<s<t .
\end{aligned}
$$


Since $\omega(\cdot, s)$ is bounded in $L^{1}\left(\mathbf{R}^{2}\right)$, it follows from (2.3) that the second integral in the right-hand side converges to zero as $s$ goes to zero. On the other hand, since $y \mapsto \Gamma_{u}(x, t ; y, 0)$ is continuous and vanishes at infinity, and since $\omega(\cdot, s) \rightarrow \alpha \delta_{0}$ as $s \rightarrow 0$, we can take the limit $s \rightarrow 0$ in the first integral and we obtain the useful representation:

$$
\omega(x, t)=\alpha \Gamma_{u}(x, t ; 0,0), \quad x \in \mathbf{R}^{2}, \quad t>0 .
$$

This formula shows in particular that $\omega \equiv 0$ if $\alpha=0$. Thus, upon replacing $\omega\left(x_{1}, x_{2}, t\right)$ with $-\omega\left(x_{2}, x_{1}, t\right)$ if needed, we can assume from now on that $\alpha>0$. To simplify the notation, we denote by $\Omega(x, t)$ the Oseen vortex with circulation $\alpha$, namely

$$
\Omega(x, t)=\frac{\alpha}{4 \pi t} \mathrm{e}^{-\frac{|x|^{2}}{4 t}}, \quad x \in \mathbf{R}^{2}, \quad t>0 .
$$

From (2.2), (2.5) we have

$$
0<\omega(x, t) \leq \frac{K_{1} \alpha}{t} \mathrm{e}^{-\beta \frac{|x|^{2}}{4 t}}, \quad x \in \mathbf{R}^{2}, \quad t>0
$$

Moreover, in view of (2.4),

$$
\int_{\mathbf{R}^{2}} \omega(x, t) \mathrm{d} x=\alpha=\int_{\mathbf{R}^{2}} \Omega(x, t) \mathrm{d} x, \quad t>0 .
$$

In particular $\|\omega(\cdot, t)\|_{L^{1}}=\alpha$ for all $t>0$, so that we can take $K=|\alpha|$ in the statement of Theorem 1.1 without loss of generality. Finally, using (1.1) and integrating by parts, we find

$$
\frac{\mathrm{d}}{\mathrm{d} t} \int_{\mathbf{R}^{2}}|x|^{2} \omega \mathrm{d} x=\int_{\mathbf{R}^{2}}|x|^{2}(\Delta \omega-u \cdot \nabla \omega) \mathrm{d} x=4 \alpha+2 \int_{\mathbf{R}^{2}}(x \cdot u) \omega \mathrm{d} x .
$$

Actually, the last integral vanishes identically. This can be seen by replacing $u$ with its expression (1.2) and using the symmetry properties of the Biot-Savart kernel. Thus we find that $\frac{\mathrm{d}}{\mathrm{d} t} \int_{\mathbf{R}^{2}}|x|^{2} \omega \mathrm{d} x=4 \alpha$, and in view of (2.7) we conclude that

$$
\int_{\mathbf{R}^{2}}|x|^{2} \omega(x, t) \mathrm{d} x=4 \alpha t=\int_{\mathbf{R}^{2}}|x|^{2} \Omega(x, t) \mathrm{d} x, \quad t>0 .
$$

\section{$3 \quad$ Uniqueness proof using entropy estimates}

We first introduce the entropy functionals that will be used in the proof. Let $f: \mathbf{R}^{2} \rightarrow(0,+\infty)$ be a $C^{1}$ function satisfying $\int_{\mathbf{R}^{2}} f(\xi) \mathrm{d} \xi=1$. We define

$$
H(f)=\int_{\mathbf{R}^{2}} f(\xi) \log \left(\frac{f(\xi)}{G(\xi)}\right) \mathrm{d} \xi, \quad I(f)=\int_{\mathbf{R}^{2}} f(\xi)\left|\nabla \log \left(\frac{f(\xi)}{G(\xi)}\right)\right|^{2} \mathrm{~d} \xi,
$$

where $G$ is as in (1.5). In kinetic theory, $H(f)$ is the relative Boltzmann entropy of the distribution function $f$ with respect to the Gaussian $G$. In information theory, $H(f)$ is often called the relative Kullback entropy of $f$ with respect to $G$, and $I(f)$ the relative Fisher information of $f$ with respect to $G$. The entropy $H(f)$ satisfies the following bounds (see for instance [4])

$$
\frac{1}{2}\|f-G\|_{L^{1}}^{2} \leq H(f) \leq I(f)
$$

where the first inequality is the Csiszár-Kullback inequality and the second bound is the StamGross logarithmic Sobolev inequality. It follows in particular from (3.2) that $H(f) \geq 0$, and $H(f)=0$ if and only if $f=G$. 
Now, let $\omega(x, t)$ be a solution of (1.1) satisfying the hypotheses of Theorem 1.1 and let $u(x, t)$ be the associated velocity field. As in the previous section, we assume without loss of generality that $T=+\infty$ and that $\alpha=\int_{\mathbf{R}^{2}} \omega(x, t) \mathrm{d} x>0$. Following [12, we define the rescaled vorticity $w(\xi, \tau)$ and the rescaled velocity field $v(\xi, \tau)$ by

$$
w(\xi, \tau)=\mathrm{e}^{\tau} \omega\left(\xi \mathrm{e}^{\frac{\tau}{2}}, \mathrm{e}^{\tau}\right), \quad v(\xi, \tau)=\mathrm{e}^{\frac{\tau}{2}} u\left(\xi \mathrm{e}^{\frac{\tau}{2}}, \mathrm{e}^{\tau}\right), \quad \xi \in \mathbf{R}^{2}, \quad \tau \in \mathbf{R} .
$$

The equation satisfied by $w(\xi, \tau)$ reads

$$
\partial_{\tau} w+\left(v \cdot \nabla_{\xi}\right) w=\Delta_{\xi} w+\frac{1}{2}\left(\xi \cdot \nabla_{\xi}\right) w+w .
$$

Since $\operatorname{div} v=0$ and $\partial_{1} v_{2}-\partial_{2} v_{1}=w$, the rescaled velocity $v$ is obtained from the rescaled vorticity $w$ via the usual Biot-Savart law.

Our aim is to show that $w(\xi, \tau)=\alpha G(\xi)$ for all $\xi \in \mathbf{R}^{2}$ and all $\tau \in \mathbf{R}$. To do that, we consider the relative entropy $h(\tau)=H(w(\cdot, \tau) / \alpha)$, where $H$ is defined in (3.1). Since by (2.7) we have $0<w(\xi, \tau) \leq K_{1} \alpha \mathrm{e}^{-\beta|\xi|^{2} / 4}$, there exists $K_{3}>0$ such that $0 \leq h(\tau) \leq K_{3}$ for all $\tau \in \mathbf{R}$. On the other hand, using (3.4) and integrating by parts, it is not difficult to verify that

$$
\frac{\mathrm{d}}{\mathrm{d} \tau} H\left(\frac{w(\cdot, \tau)}{\alpha}\right)=-I\left(\frac{w(\cdot, \tau)}{\alpha}\right), \quad \tau \in \mathbf{R},
$$

see [12]. In view of the second inequality in (3.2), this implies that $h^{\prime}(\tau) \leq-h(\tau)$ for all $\tau \in \mathbf{R}$. Thus

$$
h(\tau) \leq \mathrm{e}^{-\left(\tau-\tau_{0}\right)} h\left(\tau_{0}\right) \leq K_{3} \mathrm{e}^{-\left(\tau-\tau_{0}\right)} \quad \text { whenever } \tau \geq \tau_{0} .
$$

Letting $\tau_{0} \rightarrow-\infty$, we obtain $h(\tau)=0$ for all $\tau \in \mathbf{R}$, hence $w(\xi, \tau)=\alpha G(\xi)$ for all $\xi \in \mathbf{R}^{2}$ and all $\tau \in \mathbf{R}$. This is the desired result.

\section{Symmetric rearrangements and applications}

In this section, we recall the definition of the symmetric nonincreasing rearrangement of a function $f: \mathbf{R}^{N} \rightarrow \mathbf{R}$, and we establish two results that will be used in the final section. We refer to [1], [5], 16] for more information on rearrangements and their applications.

Let $f: \mathbf{R}^{N} \rightarrow \mathbf{R}$ be a measurable function. We assume that $f$ vanishes at infinity in the sense that meas $\left(\left\{x \in \mathbf{R}^{N}|| f(x) \mid>t\right\}\right)<\infty$ for all $t>0$. We define the distribution function $\mu_{f}:[0,+\infty) \rightarrow[0,+\infty]$ by

$$
\mu_{f}(t)=\operatorname{meas}\left(\left\{x \in \mathbf{R}^{N}|| f(x) \mid>t\right\}\right), \quad t \geq 0,
$$

and the nonincreasing rearrangement $f^{*}:[0,+\infty) \rightarrow[0,+\infty]$ by

$$
f^{*}(s)=\sup \left\{t \geq 0 \mid \mu_{f}(t)>s\right\}, \quad s \geq 0 .
$$

The symmetric nonincreasing rearrangement $f^{\#}: \mathbf{R}^{N} \rightarrow[0,+\infty]$ is then defined by

$$
f^{\#}(x)=f^{*}\left(c_{N}|x|^{N}\right), \quad x \in \mathbf{R}^{N},
$$

where $c_{N}=\pi^{N / 2} / \Gamma\left(\frac{N}{2}+1\right)$ is the measure of the unit ball in $\mathbf{R}^{N}$. By construction, $f \#$ is radially symmetric and nonincreasing (along rays). Moreover, $f^{\#}$ is lower semicontinuous (hence measurable) and meas $\left(\left\{x \in \mathbf{R}^{N} \mid f^{\#}(x)>t\right\}\right)=\operatorname{meas}\left(\left\{x \in \mathbf{R}^{N}|| f(x) \mid>t\right\}\right)$ for all $t>0$. As a consequence, if $f \in L^{p}\left(\mathbf{R}^{N}\right)$ for some $p \in[1, \infty]$, then $f^{\#} \in L^{p}\left(\mathbf{R}^{N}\right)$ and $\left\|f^{\#}\right\|_{L^{p}}=\|f\|_{L^{p}}$. More generally, one has $\left\|f^{\#}-g^{\#}\right\|_{L^{p}} \leq\|f-g\|_{L^{p}}$ for all $f, g \in L^{p}\left(\mathbf{R}^{N}\right)$. Note also that $f^{\#}=|f|^{\#}$, and that $f^{\#}$ is a continuous function if $f$ is continuous.

We next introduce a partial order on integrable functions which is based on rearrangements. 
Definition 4.1 Let $f, g: \mathbf{R}^{N} \rightarrow \mathbf{R}$ be integrable functions. We say that $f$ is dominated by $g$ if

$$
\int_{B_{R}} f^{\#}(x) \mathrm{d} x \leq \int_{B_{R}} g^{\#}(x) \mathrm{d} x \text { for all } R>0,
$$

where $B_{R}=\left\{x \in \mathbf{R}^{N}|| x \mid<R\right\}$. In this case, we write $f \preceq g$.

It can be shown that $f \preceq g$ if and only if

$$
\int_{\mathbf{R}^{N}} \Phi(|f(x)|) \mathrm{d} x \leq \int_{\mathbf{R}^{N}} \Phi(|g(x)|) \mathrm{d} x
$$

for all convex functions $\Phi:[0,+\infty) \rightarrow[0,+\infty)$ with $\Phi(0)=0$, see [1]. In particular, if $f \preceq g$ and $g \in L^{1}\left(\mathbf{R}^{N}\right) \cap L^{\infty}\left(\mathbf{R}^{N}\right)$, then $\|f\|_{L^{p}} \leq\|g\|_{L^{p}}$ for any $p \geq 1$.

The uniqueness proof in Section 5 will be based on two properties of the domination relation which we now describe. These results are rather standard, and the proofs will be outlined for completeness only.

Proposition 4.2 Let $f, g: \mathbf{R}^{N} \rightarrow[0,+\infty)$ be continuous and integrable functions satisfying:

a) $f \preceq g$;

b) $g=g^{\#}$;

c) $\int_{\mathbf{R}^{N}} f(x) \mathrm{d} x=\int_{\mathbf{R}^{N}} g(x) \mathrm{d} x$;

d) $\int_{\mathbf{R}^{N}}|x|^{N} f(x) \mathrm{d} x=\int_{\mathbf{R}^{N}}|x|^{N} g(x) \mathrm{d} x<\infty$.

Then $f=g$.

Remark 4.3 As is clear from the proof, the same result holds if $|x|^{N}$ is replaced by $\varphi(|x|)$ in assumption d), where $\varphi$ is any continuous, positive, and strictly increasing function on $[0,+\infty)$.

Proof: We first observe that

$$
\int_{\mathbf{R}^{N}}|x|^{N} f^{\#}(x) \mathrm{d} x \leq \int_{\mathbf{R}^{N}}|x|^{N} f(x) \mathrm{d} x,
$$

and that equality holds in (4.2) if and only if $f=f^{\#}$. Indeed, this is obvious if $f$ is the characteristic function of a bounded open set $A \subset \mathbf{R}^{N}$, because then $f^{\#}=\mathbf{1}_{B_{R}}$ where $c_{N} R^{N}=$ meas $(A)$. The general case follows using the "layer cake representation" [16

$$
f(x)=\int_{0}^{\infty} \mathbf{1}_{\{f>t\}}(x) \mathrm{d} t, \quad f^{\#}(x)=\int_{0}^{\infty} \mathbf{1}_{\{f>t\}}^{\#}(x) \mathrm{d} t,
$$

together with Fubini's theorem.

Now, let $h=g^{*}-f^{*}$, where $f^{*}, g^{*}$ are the nonincreasing rearrangements of $f, g$. For all $R>0$, we have

$$
\int_{B_{R}} f^{\#}(x) \mathrm{d} x=\int_{0}^{c_{N} R^{N}} f^{*}(s) \mathrm{d} s, \quad \int_{B_{R}}|x|^{N} f^{\#}(x) \mathrm{d} x=\frac{1}{c_{N}} \int_{0}^{c_{N} R^{N}} s f^{*}(s) \mathrm{d} s .
$$

Using the first equality, we can rewrite assumptions a) and c) as follows:

a') $\int_{0}^{r} h(s) \mathrm{d} s \geq 0$ for all $r>0$,

c') $\int_{0}^{\infty} h(s) \mathrm{d} s=0$.

In view of (4.2) and the second equality, assumptions b) and d) also imply 
d') $\int_{0}^{\infty} \operatorname{sh}(s) \mathrm{d} s \geq 0$, with equality if and only if $f=f^{\#}$.

Let $\mathcal{H}(r)=\int_{0}^{r} h(s) \mathrm{d} s=-\int_{r}^{\infty} h(s) \mathrm{d} s \geq 0$. Clearly, $r \mathcal{H}(r) \leq \int_{r}^{\infty} s|h(s)| \mathrm{d} s \rightarrow 0$ as $r \rightarrow+\infty$, hence

$$
\int_{0}^{\infty} \operatorname{sh}(s) \mathrm{d} s+\int_{0}^{\infty} \mathcal{H}(s) \mathrm{d} s=\int_{0}^{\infty}(\operatorname{sh}(s)+\mathcal{H}(s)) \mathrm{d} s=\left.r \mathcal{H}(r)\right|_{r=0} ^{r=\infty}=0 .
$$

By a') and $\mathrm{d}^{\prime}$ ), this implies that $\int_{0}^{\infty} \operatorname{sh}(s) \mathrm{d} s=0$ (hence $f=f^{\#}$ ) and $\mathcal{H} \equiv 0$ (hence we have $f^{\#}=g^{\#}=g$ ). This concludes the proof.

Assume now that $f: \mathbf{R}^{N} \times[0,+\infty) \rightarrow \mathbf{R}$ is a solution of the convection-diffusion equation

$$
\partial_{t} f(x, t)+U(x, t) \cdot \nabla f(x, t)=\Delta f(x, t), \quad x \in \mathbf{R}^{N}, \quad t>0,
$$

where $U: \mathbf{R}^{N} \times[0,+\infty) \rightarrow \mathbf{R}^{N}$ is a smooth, divergence-free vector field which is bounded together with all its derivatives. Assume moreover that the initial condition $f_{0}(x)=f(x, 0)$ is continuous and decays rapidly at infinity.

Proposition 4.4 Under the assumptions above, the solution of (4.3) with initial data $f_{0}$ satisfies

$$
f(\cdot, t) \preceq \mathrm{e}^{t \Delta} f_{0}^{\#}, \quad \text { for all } t \geq 0
$$

Proof: The proof uses two main ingredients:

i) If $f \preceq g$ and $g=g^{\#}$, then $\mathrm{e}^{t \Delta} f \preceq \mathrm{e}^{t \Delta} g$ for all $t>0$, see ([3], Proposition 3). This is because the integral kernel of the heat semigroup is positive, radially symmetric, and decreasing along rays (in other words, it coincides with its own rearrangement).

ii) Let $S\left(t_{1}, t_{2}\right)$ be the evolution operator associated to the linear nonautonomous transport equation

$$
\partial_{t} f(x, t)+U(x, t) \cdot \nabla f(x, t)=0, \quad x \in \mathbf{R}^{N}, \quad t>0,
$$

so that $f\left(\cdot, t_{1}\right)=S\left(t_{1}, t_{2}\right) f\left(\cdot, t_{2}\right)$. Since $U(x, t)$ is globally Lipschitz and divergence-free, one has $S\left(t_{1}, t_{2}\right) f=f \circ \Psi\left(t_{1}, t_{2}\right)^{-1}$, where $\Psi\left(t_{1}, t_{2}\right): \mathbf{R}^{N} \rightarrow \mathbf{R}^{N}$ is a measure preserving transformation. It follows immediately that $\left(S\left(t_{1}, t_{2}\right) f\right)^{\#}=f^{\#}$.

To prove the claim, we use an approximation procedure in the spirit of Trotter's formula, see ([19], Section 11.A). Fix $t>0$. Given $n \in \mathbf{N}^{*}$, we define finite sequences $\left\{f_{k}^{n}\right\}_{k=0, \ldots, n}$ and $\left\{g_{k}^{n}\right\}_{k=0, \ldots, n}$ inductively by

$$
\begin{array}{lll}
f_{k+1}^{n}=S\left(\frac{(k+1) t}{n}, \frac{k t}{n}\right) \mathrm{e}^{\frac{t}{n} \Delta} f_{k}^{n}, & & f_{0}^{n}=f_{0}, \\
g_{k+1}^{n}=\mathrm{e}^{\frac{t}{n} \Delta} g_{k}^{n}, & g_{0}^{n}=f_{0}^{\#} .
\end{array}
$$

Using properties i) and ii) above, it is easy to verify by induction over $k$ that $f_{k}^{n} \preceq g_{k}^{n}$ for all $k \in\{0, \ldots, n\}$. In particular, $f_{n}^{n} \preceq g_{n}^{n}$. But $g_{n}^{n}=\mathrm{e}^{t \Delta} f_{0}^{\#}$, and a classical result shows that $f_{n}^{n} \rightarrow f(\cdot, t)$ in $L^{1}\left(\mathbf{R}^{N}\right)$ as $n \rightarrow \infty$, so that $\left(f_{n}^{n}\right)^{\#} \rightarrow f^{\#}(\cdot, t)$ also. Thus passing to the limit we obtain $f(\cdot, t) \preceq \mathrm{e}^{t \Delta} f_{0}^{\#}$.

\section{Uniqueness proof using symmetrization}

Let again $\omega(x, t)$ be a solution of (1.1) satisfying the hypotheses of Theorem 1.1 and let $u(x, t)$ be the associated velocity field. As usual we assume that $T=+\infty$ and that $\alpha=\int_{\mathbf{R}^{2}} \omega(x, t) \mathrm{d} x>0$. For any $t>0$, let $\omega^{\#}(x, t)$ be the symmetric nonincreasing rearrangement of $\omega(x, t)$ with respect 
to the spatial variable $x \in \mathbf{R}^{2}$. Using (2.7) and the fact that the rearrangement is order preserving, we find

$$
0<\omega^{\#}(x, t) \leq \frac{K_{1} \alpha}{t} \mathrm{e}^{-\beta \frac{|x|^{2}}{4 t}}, \quad x \in \mathbf{R}^{2}, \quad t>0 .
$$

Since $\int_{\mathbf{R}^{2}} \omega^{\#}(x, t) \mathrm{d} x=\int_{\mathbf{R}^{2}} \omega(x, t) \mathrm{d} x=\alpha$, we deduce that $\omega^{\#}(\cdot, t) \rightarrow \alpha \delta_{0}$ as $t \rightarrow 0+$.

Fix $t>s>0$. Applying Proposition 4.4 with $N=2, f\left(x, t^{\prime}\right)=\omega\left(x, t^{\prime}+s\right)$, and $U\left(x, t^{\prime}\right)=$ $u\left(x, t^{\prime}+s\right)$, we obtain

$$
\omega(\cdot, t) \preceq \mathrm{e}^{(t-s) \Delta} \omega^{\#}(\cdot, s) .
$$

As $s \rightarrow 0+$, the right-hand side converges pointwise (hence also in $L^{1}\left(\mathbf{R}^{2}\right)$ by the dominated convergence theorem) to the Oseen vortex $\Omega(\cdot, t)$ defined in (2.6). It follows that

$$
\omega(\cdot, t) \preceq \Omega(\cdot, t), \quad \text { for all } t>0 .
$$

We now fix $t>0$ and apply Proposition 4.2 with $N=2, f=\omega(\cdot, t)$, and $g=\Omega(\cdot, t)$. All assumptions are satisfied due to (2.8), (2.9), and (5.1). We conclude that $\omega(x, t)=\Omega(x, t)$ for all $x \in \mathbf{R}^{2}$, which is the desired result.

\section{References}

[1] A. Alvino, P.-L. Lions, and G. Trombetti. On optimization problems with prescribed rearrangements. Nonlinear Anal. T.M.A. 13 (1989), 185-220.

[2] A. Alvino, P.-L. Lions, and G. Trombetti. Comparison results for elliptic and parabolic equations via Schwarz symmetrization. Ann. Inst. H. Poincaré Anal. Non Linéaire 7 (1990), $37-65$.

[3] A. Alvino, P.-L. Lions, and G. Trombetti. Comparison results for elliptic and parabolic equations via symmetrization: a new approach. Differential Integral Equations 4 (1991), $25-50$.

[4] A. Arnold, P. Markowich, G. Toscani, and A. Unterreiter. On convex Sobolev inequalities and the rate of convergence to equilibrium for Fokker-Planck type equations. Comm. Partial Differential Equations 26 (2001), 43-100.

[5] C. Bandle. Isoperimetric inequalities and applications. Monographs and Studies in Mathematics 7. Pitman, London, 1980.

[6] M. Ben-Artzi. Global solutions of two-dimensional Navier-Stokes and Euler equations. Arch. Rational Mech. Anal. 128 (1994), 329-358.

[7] H. Brezis. Remarks on the preceding paper by M. Ben-Artzi: "Global solutions of twodimensional Navier-Stokes and Euler equations". Arch. Rational Mech. Anal. 128 (1994), $359-360$.

[8] E. A. Carlen and M. Loss. Optimal smoothing and decay estimates for viscously damped conservation laws, with applications to the 2-D Navier-Stokes equation. Duke Math. J. 81 (1995), 135-157 (1996).

[9] A. Carpio. Asymptotic behavior for the vorticity equations in dimensions two and three. Comm. Partial Differential Equations 19 (1994), 827-872. 
[10] G.-H. Cottet. Equations de Navier-Stokes dans le plan avec tourbillon initial mesure. C. $R$. Acad. Sci. Paris Sér. I Math. 303 (1986), 105-108.

[11] I. Gallagher and Th. Gallay. Uniqueness for the two-dimensional Navier-Stokes equation with a measure as initial vorticity. Preprint, Ecole Polytechnique, 2004. Available at http://www .arXiv.org/math. AP/0406297.

[12] Th. Gallay and C. E. Wayne. Global stability of vortex solutions of the two-dimensional Navier-Stokes equation. Comm. Math. Phys., to appear. Preprint version available at http://www.arXiv.org/math.AP/0402449.

[13] Y. Giga, T. Miyakawa, and H. Osada. Two-dimensional Navier-Stokes flow with measures as initial vorticity. Arch. Rational Mech. Anal. 104 (1988), 223-250.

[14] M.-H. Giga and Y. Giga. Nonlinear partial differential equations - asymptotic behaviour of solutions and self-similar solutions. Book in preparation.

[15] T. Kato. The Navier-Stokes equation for an incompressible fluid in $\mathbf{R}^{2}$ with a measure as the initial vorticity. Differential Integral Equations 7 (1994), 949-966.

[16] E. Lieb and M. Loss. Analysis. Graduate Studies in Mathematics 14. American Mathematical Society, Providence, RI, 1997.

[17] H. Osada. Diffusion processes with generators of generalized divergence form. J. Math. Kyoto Univ. 27 (1987), 597-619.

[18] G. Talenti. Elliptic equations and rearrangements. Ann. Scuola Norm. Sup. Pisa 3 (1976), $697-718$.

[19] M. Taylor. Partial differential equations II. Qualitative studies of linear equations. Applied Mathematical Sciences 116, Springer, New-York, 1996. 